\title{
Special issue in honor of Eberhard K.U. Gross for his 65th birthday ${ }^{\star}$
}

\author{
Miguel A.L. Marques ${ }^{1, a}$, Fernando Nogueira ${ }^{2}$, Angel Rubio ${ }^{3,4,5}$, and Carsten A. Ullrich ${ }^{6}$ \\ ${ }^{1}$ Institut für Physik, Martin-Luther-Universität Halle-Wittenberg, 06120 Halle, Germany \\ ${ }^{2}$ CFsUC, Department of Physics, University of Coimbra, Rua Larga, 3004-516 Coimbra, Portugal \\ ${ }^{3}$ Max Planck Institute for the Structure and Dynamics of Matter, Luruper Chaussee 149, 22761 Hamburg, Germany \\ ${ }^{4}$ Nano-Bio Spectroscopy Group, Universidad del País Vasco, 20018 San Sebastián, Spain \\ ${ }^{5}$ Center for Computational Quantum Physics (CCQ), The Flatiron Institute, New York NY 10010, USA \\ ${ }^{6}$ Department of Physics and Astronomy, University of Missouri, Columbia, MO 65211, USA
}

Received 16 August 2018

Published online 28 November 2018

(C) EDP Sciences / Società Italiana di Fisica / Springer-Verlag GmbH Germany, part of Springer Nature, 2018

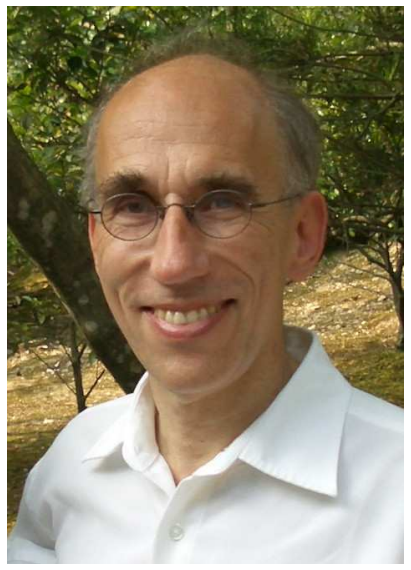

Fig. 1. Hardy Gross in Japan in 2007.

\section{Introduction}

With this special issue of the European Physical Journal B we pay homage to the scientific career of Eberhard Kurt Ulrich (Hardy) Gross (see Fig. 1), on occasion of his 65th birthday. Hardy is one of the most influential researchers in the field of theoretical density functional theory (DFT). His significant contributions started early, already as a student of Reiner Dreizler in Frankfurt and as a post-doc with the Nobel prize laureate Walter Kohn. In those years, Hardy Gross contributed to the birth of timedependent density functional theory (TDDFT), DFT for superconductors, ensemble DFT, etc. Later, he was interested in other topics of electronic structure theory. This issue contains original contributions with topics close to

\footnotetext{
${ }^{\star}$ Contribution to the Topical Issue "Special issue in honor of Hardy Gross", edited by C.A. Ullrich, F.M.S. Nogueira, A. Rubio, and M.A.L. Marques.

a e-mail: marques@tddft.org
}

Hardy's heart (some of them already mentioned above), and is a mixture of colloquium and research papers.

\section{Scientific career}

Hardy Gross was born in Frankfurt am Main in 1953. After finishing high school, where he won a first prize in the German national mathematics competition in 1971, he began to study mathematics and physics at the Johann Wolfgang Goethe University in Frankfurt in 1972. He received his $\mathrm{PhD}$ in physics in 1980 under the supervision of Professor Reiner M. Dreizler. His PhD thesis was entitled "Der Dichtefunktional-Formalismus für Atome und quasimolekulare Zweizentren-Systeme und seine relativistische Erweiterung" (density-functional formalism for atoms and quasi-molecular two-center systems and its relativistic extension).

In the late $70 \mathrm{~s}$ and early $80 \mathrm{~s}$, the field of DFT was very different from what it is today: nuclear physicists attended DFT conferences, usage in chemistry was scarce, and essentially all applications in solid-state physics relied on the local-density approximation (LDA). Systematic gradient corrections to the LDA were seen by many as the natural path forward (in the 90s, this idea was abandoned in favor of the generalized gradient approximations, or GGAs). Hardy's PhD thesis focused on deriving gradient expansions of the kinetic and exchange energy functionals using the Kirzhnits method, and developing a relativistic generalization of the Thomas-FermiDirac-Weizsäcker formalism, including detailed numerical solutions for atoms and diatomic molecules.

Besides the fundamental framework of DFT, the main interest of the Frankfurt group was in nuclear physics and atomic scattering problems. It was therefore quite natural for Hardy to start thinking about the time-dependent case, having in mind the scenario of describing a projectile colliding with an atomic target. In the period of 
1980-1984 following his PhD, Hardy remained at Frankfurt University as a research assistant, and started working with Erich Runge, a diploma student, on establishing the fundamental existence proof of TDDFT, now known as the Runge-Gross theorem [1]. During that time, Hardy also had various teaching duties; his pedagogical lectures on many-particle theory, given as a two-semester course in $1982 / 3$, were published as a textbook in 1986, again together with Erich Runge (the English version, with additional input by Olle Heinonen, appeared in 1991) [2,3].

When Walter Kohn heard Hardy give a presentation on his work on TDDFT in Portugal, he was intrigued and invited him to come to the University of California in Santa Barbara. Hardy then spent the next 6 years, from 1984 to 1990, as Heisenberg fellow in Santa Barbara (and eventually succeeded in convincing Walter that the Runge-Gross theorem was correct!). During this highly productive period, Hardy and Walter carried out seminal work in TDDFT in the linear-response regime, DFT for superconductors, and ensemble-DFT for calculating excited states (the latter two projects also involving Luiz Oliveira).

1990 was then the year of Hardy's return to Germany. He received a call to the Institute of Theoretical Physics at the University of Würzburg, an hour and a half down the A3 autobahn from Frankfurt, where he remained as a professor until 2001. In Würzburg he began to establish himself as one of the worldwide leading figures in the field of DFT, especially after the publication of his DFT book, which was written together with Reiner Dreizler [4]. In the following decade, Hardy and his group laid the foundations for using TDDFT as a practical method to calculate molecular excitation energies using a response formalism [5] (which is now widely used in all quantum chemistry and material science codes dealing with linear and nonlinear electronic properties), and for exploring the nonlinear physics of atoms and molecules in strong laser fields. The early scientific work up to this point of Hardy Gross is discussed in [6].

In 2001, Hardy and his group moved to Berlin, where he became a professor of theoretical physics at the Free University, and was elected Max-Planck-Fellow at the Fritz Haber Institute. In his years in Berlin, Hardy broadened his research scope and worked on a wide range of DFT-related topics such as combining (TD)DFT with optimal control theory, TDDFT for open quantum systems, reduced density matrix functional theory, or static and time-dependent quantum transport. In particular, Hardy's almost two-decade-long efforts to develop an abinitio DFT-based theory of superconductivity were now bearing fruit, leading to a series of papers with impressive results for various superconducting materials.

In the early 2000s, it was clear that the field of TDDFT needed to have a specific meeting to teach students and researchers entering the field the basics of the theory and the exciting problems being tackled with it. In this spirit, we started to organize with Hardy what has now become the biennial school and workshop "Time-Dependent Density-Functional Theory: Prospects and Applications", held at the beautiful Benasque Center for Science in the Spanish Pyrenees for two weeks. The first meeting was in 2004 and in the following years it led to two of the main reference books in the field $[7,8]$. Many nice discussions, new ideas and joint adventures and, above all, many great memories from Hardy!

As Hardy's international reputation continued to grow, he took on many duties and responsibilities within the scientific community. He was active as node coordinator in several European research networks, in particular the Psi-k network, served as president of the CECAM council (Centre Européen du Calcul Atomique et Moléculaire) from 2004 to 2008, and became a member of several international editorial and advisory boards.

In 2009, Hardy was appointed Director of the Theory Department at the Max Planck Institute of Microstructure Physics in Halle, where he still is today. His research team is working on the development and application of a broad range of theoretical and computational methods to describe the electronic, optical and magnetic properties of matter, including nonadiabatic nuclear dynamics, most of them, but not all, based on DFT and TDDFT. In July 2017, he was appointed Professor of Chemistry at the Hebrew University in Jerusalem.

Among the numerous honors and awards that Hardy has received are the Senior CMOA Medal for outstanding scientific achievements in 2015, the Tsungming Tu Prize (Taiwan Ministery of Science and Technology) in 2016, the Berni Alder CECAM Prize in 2016, he was elected Fellow of the American Physical Society in 2017, and he was recently awarded one of the prestigious European Research Council Advanced Grants.

\section{This special issue}

The articles collected in this issue cover a wide and impressive range of topics in the fields of chemistry, physics and material science, all of them related to Hardy's research interests. Most of the authors are his former students and postdocs or his former and present collaborators.

Although Hardy Gross is famous for his work in timedependent DFT, he also gave substantial contributions to the field of static, time-independent, DFT. Here, we should remember the book Density Functional Theory: An Approach to the Quantum Many-Body Problem [4], co-authored with Reiner Dreizler, which helped to educate several generations of "density functionalists". Several contributions to this volume revolve around standard DFT. Perdew and Ruzsinszky explain in detail the famous band-gap problem of DFT [9], while Trushin and Görling review several methods (exchange-only, hybrid functionals, and many-body GW) to calculate this quantity [10]. The response part of the exchange-correlation potential, a quantity that plays a very important role, for example for the calculation of accurate band gaps and excitation energies, is analyzed in [11] in the strongly interacting limit. Callow and Gidopoulos discuss the optimal power series expansions of the Kohn-Sham potential, and in particular the different choices for the zeroth-order Hamiltonian in such expansions [12]. An interesting alternative to the evaluation of the Hartree and exchange-correlation potential of a finite $N$-electron 
system is to replaced it by the electrostatic potential of an effective charge density, a method whose performance is analyzed in [13]. Gómez Pueyo and Castro discuss the relation of electron-electron interaction potentials with exchange and correlation functionals [14], while Takada looks at the on-top density in the nonlinear metallic screening and its implication on the correlation functional [15]. In some cases, the highest-occupied molecular orbital has a nodal plane that extends to infinity, leading to different cases for the asymptotic behavior of the exact density and of the exact Kohn-Sham potential. The consequences for the potential in the effective equation for the square root of the density are studied in [16]. Resta discusses the modern theory of polarization - based on a Berry phase - and in particular the relation between the Kohn-Sham polarization and the real one [17]. Oueis and Wasserman obtain numerically the exact partition potential for a 1D system of interacting electrons designed to model lithium hydride, highlighting the importance of non-additive noninteracting kinetic energy to the exchange-correlation [18]. Petrone et al. describe an efficient implementation of two-component relativistic DFT with torque-free auxiliary variables [19]. Finally, there are two applications of DFT to real materials, namely to the metastable $(2 \times 2)$ reconstruction of barium on graphene [20] and to $\mathrm{Cu}-\mathrm{Au}$ intermetallic compounds [21].

TDDFT has a special place in this Special Issue and in the work of Hardy Gross. The derivation of the by now famous Runge-Gross theorem [1] opened the door to the study of many-body electronic systems under the influence of a time-dependent field. It is not surprising, therefore, that a large number of contributions to this volume revolve around this theory. Nielsen et al. discuss the numerical construction of the Runge-Gross mapping between time-dependent densities and potentials [22]. In [23], the authors provide a rigorous basis for the use of non-multiplicative potentials in TDDFT, the use of which is outside original Kohn-Sham theory. In [24], Nagy develops a time-dependent functional theory that uses the density of electron pairs as basic variable. The problem of $v_{0}$ representability in lattice ensemble-DFT and its signature in lattice TDDFT is presented in [25]. Sangalli et al. use non-equilibrium many-body perturbation theory, a powerful alternative to TDDFT, to study coherent and non-coherent exciton dynamics [26]. In [27], non-equilibrium Green's functions are compared against configuration interaction techniques for time-dependent Auger decay processes. Umerbekova et al. look at the renormalization of energy levels and the enhancement of polarizability of molecules at surfaces with subsystem TDDFT [28]. In [29] Ahlert et al. present an iterative approach for the moment representation of the densitydensity response function. Finally, Dinh et al. discuss extensions of time-dependent theories in order to include incoherent dynamical correlations [30].

Of course, and as Hardy often states, the usefulness of a DFT depends on the existence of good exchange-correlation functionals. Several articles in this Special Issue look at this problem. The finite-temperature extension of TDDFT is discussed in [31], where also an approximation for the exchange-correlation kernel is obtained from the finite-temperature generalization of the retarded cumulant expansion applied to the homogeneous electron gas. Hellgren writes an expression for the derivative discontinuity in terms of the exchange-correlation kernel and demonstrates the crucial role of a discontinuity in the kernel itself [32]. In [33], the response obtained with the exchange approximation by Armiento and Kümmel [34] and by Becke [35], both of which show divergences on orbital nodal planes, is closely investigated. Liao et al. perform non-adiabatic calculations for a one-dimensional two-electron Helium model in a triplet state using the recently formulated Sturm-Liouville-type equation for the time-dependent optimized effective potential with the exact exchange functional [36]. In [37], van Meer and Gritsenko discuss bond-breaking excitations from functionals at the highest rung of Jacob's ladder, i.e., functionals in which both occupied and virtual Kohn-Sham orbitals participate. Finally, Lacombe et al. discuss the influence of exchange-correlation functionals in the study of electron scattering problems [38].

The time-dependent Kohn-Sham equations describe the evolution of the electrons in the presence of a timedependent potential. As such, the direct solution of this equation in the time-domain gives access to all nonlinear and the linear response properties of the system. In fact, even within the linear response regime it is often more favorable to solve the equations in time, as this method has a better scaling than the solution of the equations in the frequency domain. Nowadays a series of codes can perform these real-time simulations out of the box. We should also remember that Hardy contributed with ideas, resources, and enthusiasm, to the initial development of octopus [39], perhaps the first large-scale effort to develop a real-time TDDFT code. In this Special Issue, we can find several interesting articles that use real-time simulations. In [40] electron dynamics induced by short laser pulses on a gold surface are investigated, while Niehaus et al. look at how pulse shape and molecular orientation determine the attosecond charge migration in caffeine [41]. The calculation of electronic stopping power within a Gaussian basis set description is described in [42], while electronic stopping and proton dynamics in III-IV semiconductors is studied in [43]. Real-time TDDFT can also be used to calculate attosecond photoelectron spectroscopy as shown in [44]. Finally, Andrade et al. calculate the negative differential conductivity in liquid aluminum from real-time quantum simulations [45].

One of the most useful applications of TDDFT in the linear regime is for the calculation of electronic excitation energies and the closely related optical absorption spectra. In fact, some of the first applications of TDDFT to the calculation of these properties were performed by Hardy's group [5,46] as early as 1996. There are several contributions in this Special Issue regarding the calculation of optical spectra using TDDFT and other approaches. Ferradas et al. use time-dependent currentdensity functional theory to obtain the optical properties of alkali metals [47]. Cardoso et al. look at the optical spectra of graphene nanoribbons [48], while in [49] the object of the research are six isomers of a $\pi$-conjugated 
carbon nanocage. The optical properties of quantum dots of $\mathrm{Cu}_{2} \mathrm{ZnSn}(\mathrm{Se}, \mathrm{S})_{4}$, a material currently investigated for its use as an absorber in photovoltaic cells, is investigated in [50]. In [51], the authors propose a way to tune the electronic and optical properties of the $\mathrm{Ba}-\mathrm{Zr}-\mathrm{S}$ system via dimensional reduction. Finally, Nazarov looks at the collective and independent-particle excitations in quasi-2D electron gas with one filled miniband [52].

There are a couple of contributions to this Special Issue regarding visualization of physical concepts. We remember that, in this context, Hardy introduced the time-dependent electron-localization function in 2005 [53], that allows for the visualization of the dynamics of bonds, lone-pairs, ionization, etc. Pluhar and Ullrich discuss how to visualize electronic excitations with the particle-hole map [54], while Pittalis et al. show how to measure electron localization by means of coalescent pairs [55].

A topic that has interested Hardy already for a few years is reduced density-matrix functional theory. Hardy's group has come up with a number of interesting functionals $[56,57]$ and has obtained many interesting results, in particular for solids [58]. In this volume, we find two contributions proposing new functionals, one from manybody perturbation theory [59], and the second using the generalized Pauli principle [60]. Mitxelena et al. look at the phase dilemma in constructing such functionals [61]. Gebauer et al. discuss the related approach of occupationprobability-based, natural-spin-orbital functional theories [62]. Finally, the natural orbitals of a spin-singlet helium atom in a linearly polarized laser field are calculated and discussed in [63] for a high-harmonic-generation process.

The field of DFT for superconductors was born with the seminal paper of Oliveira, Gross, and Kohn in 1988 [64], and Hardy has been its main driving force since then. A major landmark was the development of working exchange-correlation functionals $[65,66]$ for both the electron-electron and electron-phonon interaction and the application of the theory to several high-profile problems $[67,68]$. In this issue, we find three articles on this subject, a study of superconductivity in doped polyethylene at high pressures [69], an investigation of hydrogenated carbon nanostructures [70], and finally a description of the nonunitary triplet pairing in $\mathrm{LaNiC}_{2}$ [71].

Another topic that has interested Hardy for many years is the interaction between electrons [72] and nuclei, how to go beyond the Born-Oppenheimer approximation [73], and, more recently, the so-called exactfactorization of the electron-ion wave-function [74]. There are several articles in this Issue related to these problems. Gonze et al. [75] explore variations of the exact factorization ideas for arbitrary many-body Hamiltonians, while Agostini et al. [76] study nuclear quantum effects in electronic (non)adiabatic dynamics. The problem of conical intersections [77,78] and of quantumclassical approaches to excited-state dynamics [79] are also discussed from the perspective of beyond BornOppenheimer dynamics. Curchod et al. present a coupledtrajectory mixed quantum/classical method including nonadiabatic quantum coherence effects [80]. Finally, two articles $[81,82]$ discuss the exact-factorization for electron and photons.
Spin and magnetic properties form an important field of physics, and it is therefore not surprising that Hardy looked into several problems related to this area during his career. This included the development of new functionals for spin-DFT [83], the study of non-collinear magnetism $[84,85]$ and spin dynamics $[86,87]$, etc. A series of articles in this issue deal with spin. Dewhurst et al. study the effect of the exchange-correlation spin-torque on spin dynamics [88], while Gorni et al. look at spin-dynamics from time-dependent density-functional perturbation theory [89]. The theoretical exploration of a laser-driven, spin active, split-ring resonator as a source for high harmonic generation in the $\mathrm{THz}$ regime can be found in [90], while the integer quantum Hall effect in the strict $2 \mathrm{D}$ limit is discussed in [91]. In [92] a simple procedure to improve the magnon dispersion by taking into account gradient corrections to the ALDA at the exchange-only level is suggested. Two articles are concerned with new topological materials: Göbel et al. discuss the family of topological Hall effects for electrons in skyrmion crystals [93], while Vergniory et al. study magnetic Weyl fermions in PtFeSb [94]. The transmission of the spin-spin coupling constants through hydrogen bonds in ammonia clusters is the subject of [95], while the spin-state energetics of iron(II) porphyrin is discussed in [96]. Finally, Romero and Verstraete benchmark different exchange-correlation functionals for two families of binary iron alloys which are metallic and magnetic [97].

Model Hamiltonians play a very important role in physics not only to understand complex phenomena, but also as a test-bench for ab initio theories. It is therefore not surprising to find several articles in this issue pertaining to models. Four of these revolve around the Hubbard model, and in particular the Hubbard dimer: Vanzini et al. discuss the spectroscopy of this system [98], the asymmetric dimer is used to study the density-dependence of the exact frequency-dependent kernel of linear-response TDDFT [99], and the melting of this system is studied in [100] with the help of the adiabatic LDA. Finally, Deur et al. use the Hubbard dimer to explore weight-dependent density-functional approximations for ensembles [101], a theory originally developed by Gross, Oliveira, and Kohn to study excited states [102-104]. The Anderson model is used in [105], where it is discussed how sharply this model depicts a single-electron transistor.

Electronic transport can be seen as a special timedependent problem where an electronic current flows through a finite system connected to two (semi-infinite) leads. The seminal application of TDDFT to this problem included the contribution of Hardy Gross [106], which led to further investigations of the effect of bound-states in quantum transport [107], real-time switching between different steady-states [108], etc. In this special issue, exchange-correlation potentials for steady-state DFT are studied for the out-of-equilibrium Anderson model [109] and for geometries with asymmetrically coupled leads [110].

There are a number of other topics covered in this Special Issue, most of them of interest to Hardy at one moment or another in his career. Schaupp et al. show that it is possible to calculate the time-dependent electron momenta from Born-Oppenheimer calculations [111]. In 
[112], Pronobis et al. discuss a very recent approach to the study of quantum systems, namely machine learning, and how it can be used to capture intensive and extensive DFT/TDDFT molecular properties. Basilewitsch et al. combine a quantum dynamical propagator that explicitly accounts for quantum mechanical time ordering with optimal control theory, and apply it to superconducting qubits [113]. In [114], a screened independent atom model is presented for the description of ion collisions from atomic and molecular clusters. Musso et al. apply second-generation Car-Parrinello molecular dynamics techniques to the h-BN/Rh(111) nanomesh [115]. Finally, Ruotsalainen et al. discuss the isotropic Compton profile difference across the phase transition of $\mathrm{VO}_{2}[116]$.

\section{Conclusions}

We hope that Hardy enjoys reading this Special Issue, and that he is not scared away by its thickness. To finish, we all would like to join together in wishing Hardy a happy 65 th birthday, and many more years of doing Science!

We are indebted to Angelica Zacarias for providing the photograph of Hardy.

\section{References}

1. E. Runge, E.K.U. Gross, Phys. Rev. Lett. 52, 997 (1984)

2. E.K.U. Gross, E. Runge, Vielteilchentheory (Teubner, Stuttgart, 1986)

3. E.K.U. Gross, E. Runge, O. Heinonen, Many-Particle Theory (Taylor and Francis, New York, 1991)

4. R.M. Dreizler, E.K.U. Gross, Density Functional Theory: an Approach to the Quantum Many-Body Problem (Springer, Berlin, 1990)

5. M. Petersilka, U.J. Gossmann, E.K.U. Gross, Phys. Rev. Lett. 76, 1212 (1996)

6. C. Fiolhais, Eur. Phys. J. B 91, 250 (2018)

7. M.A.L. Marques, C.A. Ullrich, F. Nogueira, A. Rubio, K. Burke, E.K.U. Gross (Eds.), in Time Dependent Density Functional Theory, Lecture Notes in Physics (Springer, Berlin, 2006), Vol. 706

8. M.A.L. Marques, N. Maitra, F. Nogueira, E.K.U. Gross, A. Rubio (Eds.), in Fundamentals of Time-Dependent Density Functional Theory, Lecture Notes in Physics (Springer, Berlin, 2012), Vol. 837

9. J.P. Perdew, A. Ruzsinszky, Eur. Phys. J. B 91, 108 (2018)

10. E. Trushin, A. Görling, Eur. Phys. J. B 91, 149 (2018)

11. S. Giarrusso, P. Gori-Giorgi, K.J.H. Giesbertz, Eur. Phys. J. B 91, 186 (2018)

12. T.J. Callow, N.I. Gidopoulos, Eur. Phys. J. B 91, 209 (2018)

13. T. Pitts, N.I. Gidopoulos, N.N. Lathiotakis, Eur. Phys. J. B 91, 130 (2018)

14. A.G. Pueyo, A. Castro, Eur. Phys. J. B 91, 105 (2018)

15. Y. Takada, Eur. Phys. J. B 91, 189 (2018)

16. P. Gori-Giorgi, E.J. Baerends, Eur. Phys. J. B 91, 160 (2018)

17. R. Resta, Eur. Phys. J. B 91, 100 (2018)

18. Y. Oueis, A. Wasserman, Eur. Phys. J. B 91, 247 (2018)
19. A. Petrone, D.B. Williams-Young, S. Sun, T.F. Stetina, X. Li, Eur. Phys. J. B 91, 169 (2018)

20. C. Tresca, N.I. Verbitskiy, A. Grüneis, G. Profeta, Eur. Phys. J. B 91, 165 (2018)

21. H. Levämäki, L. Tian, K. Kokko, L. Vitos, Eur. Phys. J. B 91, 128 (2018)

22. S.E.B. Nielsen, M. Ruggenthaler, R. van Leeuwen, Eur. Phys. J. B 91, 235 (2018)

23. R. Baer, L. Kronik, Eur. Phys. J. B 91, 170 (2018)

24. A. Nagy, Eur. Phys. J. B 91, 110 (2018)

25. T. Rössler, C. Verdozzia, C.-O. Almbladh, Eur. Phys. J. B 91, 219 (2018)

26. D. Sangalli, E. Perfetto, G. Stefanucci, A. Marini, Eur. Phys. J. B 91, 171 (2018)

27. F. Covito, E. Perfetto, A. Rubio, G. Stefanucci, Eur. Phys. J. B 91, 216 (2018)

28. A. Umerbekova, S.-F. Zhanga, P. Sudheer Kumar, M. Pavanello, Eur. Phys. J. B 91, 214 (2018)

29. P. Ahlert, A. Scherrer, C. Dressler, D. Sebastiani, Eur. Phys. J. B 91, 94 (2018)

30. P.M. Dinh, L. Lacombe, P.-G. Reinhard, É. Suraud, M. Vincendon, Eur. Phys. J. B 91, 246 (2018)

31. J.J. Rehr, J.J. Kas, Eur. Phys. J. B 91, 153 (2018)

32. M. Hellgren, Eur. Phys. J. B 91, 155 (2018)

33. J. Garhammer, F. Hofmann, R. Armiento, S. Kümmel, Eur. Phys. J. B 91, 159 (2018)

34. R. Armiento, S. Kümmel, Phys. Rev. Lett. 111, 036402 (2013)

35. A.D. Becke, Phys. Rev. A 38, 3098 (1988)

36. S.-L. Liao, T.-S. Ho, H. Rabitz, S.-I. Chu, Eur. Phys. J. B 91, 147 (2018)

37. R. van Meer, O.V. Gritsenko, Eur. Phys. J. B 91, 122 (2018)

38. L. Lacombe, Y. Suzuki, K. Watanabe, N.T. Maitra, Eur. Phys. J. B 91, 96 (2018)

39. A. Castro, H. Appel, M. Oliveira, C.A. Rozzi, X. Andrade, F. Lorenzen, M.A.L. Marques, E.K.U. Gross, A. Rubio, Phys. Status Solidi B 243, 2465 (2006)

40. Y. Miyamoto, Eur. Phys. J. B 91, 228 (2018)

41. T.A. Niehaus, M. Meziane, F. Lepine, A. Marciniak, K. Yamazaki, H. Kono, Eur. Phys. J. B 91, 152 (2018)

42. I. Maliyov, J.-P. Crocombette, F. Bruneval, Eur. Phys. J. B 91, 172 (2018)

43. C.-W. Lee, A. Schleife, Eur. Phys. J. B 91, 222 (2018)

44. S.A. Sato, H. Hübener, A. Rubio, U. De Giovannini, Eur. Phys. J. B 91, 126 (2018)

45. X. Andrade, S. Hamel, A.A. Correa, Eur. Phys. J. B 91, 229 (2018)

46. E.K.U. Gross, J.F. Dobson, M. Petersilka, Density functional theory of time-dependent phenomena, in Density Functional Theory II: Relativistic and Time Dependent Extensions, edited by R.F. Nalewajski (Springer Berlin Heidelberg, Berlin, Heidelberg, 1996), pp. 81-172

47. R. Ferradás, J.A. Berger, P. Romaniello, Eur. Phys. J. B 91, 119 (2018)

48. C. Cardoso, A. Ferretti, D. Prezzi, Eur. Phys. J. B 91, 286 (2008)

49. Y. Noguchi, D. Hirose, O. Sugino, Eur. Phys. J. B 91, 125 (2018)

50. S. Körbel, P. Boulanger, X. Blase, M.A.L. Marques, S. Botti, Eur. Phys. J. B 91, 215 (2018)

51. Y. Li, D.J. Singh, Eur. Phys. J. B 91, 188 (2018)

52. V.U. Nazarov, Eur. Phys. J. B 91, 95 (2018)

53. T. Burnus, M.A.L. Marques, E.K.U. Gross, Phys. Rev. A 71, 010501 (2005)

54. E.A. Pluhar III, C.A. Ullrich, Eur. Phys. J. B 91, 137 (2018) 
55. S. Pittalis, D. Varsano, A. Delgado, C.A. Rozzi, Eur. Phys. J. B 91, 187 (2018)

56. S. Sharma, J.K. Dewhurst, N.N. Lathiotakis, E.K.U. Gross, Phys. Rev. B 78, 201103 (2008)

57. N.N. Lathiotakis, N. Helbig, E.K.U. Gross, Phys. Rev. B 75, 195120 (2007)

58. S. Sharma, J.K. Dewhurst, S. Shallcross, E.K.U. Gross, Phys. Rev. Lett. 110, 116403 (2013)

59. K.J.H. Giesbertz, A.-M. Uimonen, R. van Leeuwen, Eur. Phys. J. B 91, 282 (2018)

60. C.L. Benavides-Riverosa, M.A.L. Marques, Eur. Phys. J. B 91, 133 (2018)

61. I. Mitxelena, M. Rodriguez-Mayorga, M. Piris, Eur. Phys. J. B 91, 109 (2018)

62. R. Gebauer, M.H. Cohen, R. Car, Eur. Phys. J. B 91, 244 (2018)

63. J. Rapp, D. Bauer, Eur. Phys. J. B 91, 151 (2018)

64. L.N. Oliveira, E.K.U. Gross, W. Kohn, Phys. Rev. Lett. 60, 2430 (1988)

65. M. Lüders, M.A.L. Marques, N.N. Lathiotakis, A. Floris, G. Profeta, L. Fast, A. Continenza, S. Massidda, E.K.U. Gross, Phys. Rev. B 72, 024545 (2005)

66. M.A.L. Marques, M. Lüders, N.N. Lathiotakis, G. Profeta, A. Floris, L. Fast, A. Continenza, E.K.U. Gross, S. Massidda, Phys. Rev. B 72, 024546 (2005)

67. A. Floris, G. Profeta, N.N. Lathiotakis, M. Lüders, M.A.L. Marques, C. Franchini, E.K.U. Gross, A. Continenza, S. Massidda, Phys. Rev. Lett. 94, 037004 (2005)

68. P. Cudazzo, G. Profeta, A. Sanna, A. Floris, A. Continenza, S. Massidda, E.K.U. Gross, Phys. Rev. Lett. 100, 257001 (2008)

69. J.A. Flores-Livas, M. Grau?inyt?, L. Boeri, G. Profeta, A. Sanna, Eur. Phys. J. B 91, 176 (2018)

70. A. Sanna, A. Davydov, J.K. Dewhurst, S. Sharma, J.A. Flores-Livas, Eur. Phys. J. B 91, 177 (2018)

71. G. Csire, B. Újfalussy, J.F. Annett, Eur. Phys. J. B 91, 217 (2018)

72. T. Kreibich, E.K.U. Gross, Phys. Rev. Lett. 86, 2984 (2001)

73. T. Kreibich, M. Lein, V. Engel, E.K.U. Gross, Phys. Rev. Lett. 87, 103901 (2001)

74. A. Abedi, N.T. Maitra, E.K.U. Gross, Phys. Rev. Lett. 105, 123002 (2010)

75. X. Gonze, J. Sky Zhou, L. Reining, Eur. Phys. J. B 91, 224 (2018)

76. F. Agostini, I. Tavernelli, G. Ciccotti, Eur. Phys. J. B 91, 139 (2018)

77. F. Agostini, B.F.E. Curchod, Eur. Phys. J. B 91, 141 (2018)

78. A. De Sio, F.V.d.A. Camargo, K. Winte, E. Sommer, F. Branchi, G. Cerullo, C. Lienau, Eur. Phys. J. B 91, 236 (2018)

79. F. Agostini, Eur. Phys. J. B 91, 143 (2018)

80. B.F.E. Curchod, F. Agostini, I. Tavernelli, Eur. Phys. J. B 91, 168 (2018)

81. A. Abedi, E. Khosravi, I.V. Tokatly, Eur. Phys. J. B 91, 194 (2018)

82. N.M. Hoffmann, H. Appel, A. Rubio, N.T. Maitra, Eur. Phys. J. B 91, 180 (2018)

83. K. Capelle, E.K.U. Gross, Phys. Rev. Lett. 78, 1872 (1997)

84. F.G. Eich, E.K.U. Gross, Phys. Rev. Lett. 111, 156401 (2013)
85. F.G. Eich, S. Kurth, C.R. Proetto, S. Sharma, E.K.U. Gross, Phys. Rev. B 81, 024430 (2010)

86. S. Sharma, J.K. Dewhurst, C. Ambrosch-Draxl, S. Kurth, N. Helbig, S. Pittalis, S. Shallcross, L. Nordström, E.K.U. Gross, Phys. Rev. Lett. 98, 196405 (2007)

87. P. Elliott, K. Krieger, J.K. Dewhurst, S. Sharma, E.K.U. Gross, New J. Phys. 18, 013014 (2016)

88. J.K. Dewhurst, A. Sanna, S. Sharma, Eur. Phys. J. B 91, 218 (2018)

89. T. Gorni, I. Timrov, S. Baroni, Eur. Phys. J. B 91, 249 (2018)

90. D. Schulze, J. Wätzel, J. Berakdar, Eur. Phys. J. B 91, 106 (2018)

91. D. Miravet, C.R. Proetto, Eur. Phys. J. B 91, 129 (2018)

92. F.G. Eich, S. Pittalis, G. Vignale, Eur. Phys. J. B 91, $173(2018)$

93. B. Göbel, A. Mook, J. Henk, I. Mertig, Eur. Phys. J. B 91, 179 (2018)

94. M.G. Vergniory, L. Elcoro, F. Orlandi, B. Balke, Y.-H. Chan, J. Nuss, A.P. Schnyder, L.M. Schoop, Eur. Phys. J. B 91, 213 (2018)

95. J.S. Fabián, S. Omar, J.M. García de la Vega, Eur. Phys. J. B 91, 124 (2018)

96. B. Pinter, R. Al-Saadon, Z. Chen, W. Yang, Eur. Phys. J. B 91, 270 (2018)

97. A.H. Romero, M.J. Verstraete, Eur. Phys. J. B 91, 193 (2018)

98. M. Vanzini, L. Reining, M. Gatti, Eur. Phys. J. B 91, $192(2018)$

99. D.J. Carrascal, J. Ferrer, N. Maitra, K. Burke, Eur. Phys. J. B 91, 142 (2018)

100. M. Herrera, K. Zawadzki, I. Dámico, Eur. Phys. J. B 91, 248 (2018)

101. K. Deur, L. Mazouin, B. Senjean, E. Fromager, Eur. Phys. J. B 91, 162 (2018)

102. E.K.U. Gross, L.N. Oliveira, W. Kohn, Phys. Rev. A 37, 2805 (1988)

103. E.K.U. Gross, L.N. Oliveira, W. Kohn, Phys. Rev. A 37, 2809 (1988)

104. L.N. Oliveira, E.K.U. Gross, W. Kohn, Phys. Rev. A 37, 2821 (1988)

105. K. Zawadzki, L.N. Oliveira, Eur. Phys. J. B 91, 136 (2018)

106. S. Kurth, G. Stefanucci, C.-O. Almbladh, A. Rubio, E.K.U. Gross, Phys. Rev. B 72, 035308 (2005)

107. E. Khosravi, S. Kurth, G. Stefanucci, E.K.U. Gross, Appl. Phys. A 93, 355 (2008)

108. A.-M. Uimonen, E. Khosravi, G. Stefanucci, S. Kurth, R. van Leeuwen, E.K.U. Gross, J. Phys.: Conf. Ser. 220, 012018 (2010)

109. S. Kurth, G. Stefanucci, Eur. Phys. J. B 91, 118 (2018)

110. S. Kurth, D. Jacob, Eur. Phys. J. B 91, 101 (2018)

111. T. Schaupp, J. Albert, V. Engel, Eur. Phys. J. B 91, 97 (2018)

112. W. Pronobis, K.T. Schütt, A. Tkatchenko, K.-R. Müller, Eur. Phys. J. B 91, 178 (2018)

113. D. Basilewitsch, L. Marder, C.P. Koch, Eur. Phys. J. B 91, 161 (2018)

114. H.J. Lüdde, M. Horbatsch, T. Kirchner, Eur. Phys. J. B 91, 99 (2018)

115. T. Musso, S. Caravati, J. Hutter, M. Iannuzzi, Eur. Phys. J. B 91, 148 (2018)

116. K.O. Ruotsalainen, J. Inkinen, T. Pylkkänen, T. Buslaps, M. Hakala, K. Hämäläinen, S. Huotari, Eur. Phys. J. B 91, 225 (2018) 